\title{
Advances in the genome-wide association study of chronic hepatitis B susceptibility in Asian population
}

\author{
Bing Qiu ${ }^{1 *+}$, Wei Jiang ${ }^{2 \dagger}$, Mojtaba Olyaee ${ }^{3}$, Kenji Shimura ${ }^{4}$, Akihiro Miyakawa ${ }^{4}$, Huijing Hu ${ }^{5}$, Yongcui Zhu ${ }^{1}$ \\ and Lixin Tang ${ }^{1}$
}

\begin{abstract}
Chronic hepatitis B (CHB) is the most common chronic liver disease resulting from viral infection and has become a serious threat to human health. Each year, about 1.2 million people in the world die from diseases caused by chronic infection of hepatitis B virus. The genetic polymorphism is significantly associated with the susceptibility to chronic hepatitis B. Genome-wide association study was recently developed and has become an important tool to detect susceptibility genes of CHB. To date, a number of CHB-associated susceptibility loci and regions have been identified by scientists over the world. To clearly understand the role of susceptibility loci in the occurrence of CHB is important for the early diagnosis and prevention of CHB.
\end{abstract}

Keywords: Genome-wide association study, Chronic hepatitis B, Pathogenesis, Susceptibility

\section{Background}

Chronic hepatitis $\mathrm{B}(\mathrm{CHB})$ is the most common chronic liver disease caused by viral infection and has now become a serious threat to human health. The number of chronic HBV-infected patients worldwide has so far risen to $350-400$ million, of which 25 to $40 \%$ eventually died of liver cirrhosis and liver cancer [1-3]. In China, there is a high incidence of hepatitis B with more than 120 million carriers of HBV. Each year, nearly 300,000 HBV carriers die of liver cirrhosis and liver cancer [4]. The causes of $\mathrm{CHB}$ are complicated, among which the main risk factors include familial spread, infant infection, infection due to immunologic inadequacy, and a history of other liver diseases. With advances in the epidemiological study and molecular biology research, scientists have found that many gene polymorphisms are actually associated with $\mathrm{CHB}$ occurrence [5], which has greatly enriched our understanding in the occurrence and progression of

\footnotetext{
*Correspondence: bingqiu07@163.com

${ }^{\dagger}$ Bing Qiu and Wei Jiang contributed equally to this work

1 Department of Gastroenterology, Heilongjiang Province Hospital, 82 Zhongshan Road, Harbin 150036, Heilongjiang, People's Republic of China

Full list of author information is available at the end of the article
}

CHB. In recent years, the rapid development of genomewide association study (GWAS) has become an important tool to search for CHB-related susceptibility genes. GWAS has been applied to screen CHB-related candidate SNPs by using multiple approaches of molecular biology and bioinformatics. The combined effects of important $\mathrm{CHB}$ susceptibility loci and the interaction between gene and environment have been further investigated in-depth. To clearly understand the role of each susceptibility loci in the occurrence of $\mathrm{CHB}$ will provide a solid theoretical base for early diagnosis and prevention of $\mathrm{CHB}$.

\section{Genome-wide association study}

With the completion of Human Genome Project, GWAS became possible and developed rapidly. GWAS was designed to screen the disease-related genetic markers across a complete set of genomic DNA or genome to scrutinize genetic variations or single nucleotide polymorphisms (SNPs) associated with complex diseases. The database of genotypes and phenotypes (dbGAP), an international resource sharing center, was then established to archive and distribute data from studies such as GWAS in which associations between genetic variations 
and human diseases had been identified. GWAS aims at determining genes and susceptibility loci related to the occurrence of diseases, searching for genetic markers for the disease, therefore conducting early diagnosis and effective individualized treatment, as well as developing new drugs and novel specified disease prevention. GWAS has two types of design: single-stage design and two- or multi-stage design. Single-stage design is used to genotype SNP loci in a one-time manner for all selected case-control samples, and then analyze the association between each SNP and the disease. Two- or multi-stage design is a multi-step process used to genotype all SNP loci on a genome-wide scale in a small sample of population, followed by the selection of a few SNP loci found to be most significantly associated with the studied disease after statistical analysis. In another or multiple other larger independent populations, those selected SNPs will be further genotyped, and then analyzed according to the data obtained from the previous two or multi-stage studies. Single-stage design of GWAS is a costly process due to the large sample size required for genome-wide SNP genotyping. To reduce the number of genotyping and cost, most of investigators prefer to use a two- or multistage design. The main advantage of GWAS lies in its ability to comprehensively assess the association between genetic variations and diseases on a genome-wide scale without any biological hypothesis prior to the study.

Several methods such as DNA microarray analysis, exome sequencing, whole-genome sequencing were used while performing GWAS. DNA microarray (or DNA chip) is an important adjunct in performing GWAS because it allows a single sample to be simultaneously screened for variants at $\sim 2$ million known genetic markers in a single standardized assay at the whole-genome level. However, the high-throughput nature of DNA microarray combined with tremendous amount of datasets may result in a high rate of errors [6]. The reliability and reproducibility of microarrays are generally not satisfactory, which can be resulted from a number of factors such as the complexity of human genome, the quality of DNA prepared, the specificity of probes designed, the sample size, and the methods used for statistical analysis. To improve the reliability and reproducibility of microarray to some extent, all procedures involved should be tightly regulated and stringently quality controlled. Currently, microarray-based genotyping platforms were not widely used in GWAS [7]. Exome sequencing is a transcriptomics technique used to sequence all of the expressed genes in a genome (known as the exome). It is know that human exome only consists of $1 \%$ of the human genome, indicating the cost of sequencing can be substantially lowered in exome sequencing, typically only one-sixth to whole-genome sequencing. However, exome sequencing only covers $85 \%$ of disease-related variants and the remaining $15 \%$ of variants not located within the exon will escape from the screening [8]. Moreover, it is still not clear whether exome sequencing is able to fully capture genetic variants associated with complex disease [7]. Whole-genome sequencing has become cost and time efficient with the advent of next generation sequencing (NGS). Whole-genome sequencing provides greater coverage of the entire genome, which can capture both common and rare variants. However, despite the large drop in sequencing cost for a human genome, large-scale sequencing projects are still costly, which has prevented whole-genome sequencing from being widely used in GWAS that usually require thousands of samples [9]. The quality of the resulting draft sequence from whole-genome sequencing needs to be improved. Different NGS platforms used in GWAS may produce sequencing errors ranging from 0.5 to $2 \%$ [10]. Therefore, findings from whole-genome sequencing by using NGS should be further confirmed by more validated technologies.

\section{The application of GWAS to the study of disease susceptibility}

Single nucleotide polymorphism (SNP) refers to a DNA sequence polymorphism at the genomic level, caused by only a single nucleotide variation that commonly occurs within a population. SNP is the most common genetic variation in human, accounting for over $90 \%$ of all known polymorphisms. In recent years, as the third generation of genetic markers, SNP has been widely used to search for disease-related genes and to explain the difference among individuals and groups in terms of the susceptibility to the disease and the disease progression [11]. Traditional analysis of the association between complex diseases and candidate genes usually selects one or a few disease-related candidate genes based on an understanding of the disease; therefore, genes and SNP loci for study are poorly available.

In recent years, with the completion of two projects HapMap (The International HapMap Project) and 1000 Genomes (The 1000 Genomes Project) as well as the rapid development of high-throughput genotyping technology, GWAS has become an important tool for scientists to search for disease susceptibility-related genetic loci. In 2005, a GWAS for the age-related macular degeneration was first reported in Science, which is a hallmark for the application of GWAS to the medical field [12]. GWAS is generally performed to investigate millions of SNPs across the whole-genome, and is carried out on a large-scare, multi-center basis such that associations between diseases and genes can be repeated and confirmed. GWAS is not limited to a specific gene or chromosomal region, which offers itself a great potential to 
discover unknown genes related to certain disease under investigation. It also opens a new avenue to systematically study the genetic factors associated with complex diseases. Compared with traditional candidate gene study and family linkage study, GWAS presents to be much more effective in detecting disease-related genetic loci. Currently, GWAS has become a powerful tool to study the molecular etiology of complex diseases, and has greatly advanced the understanding of human diseases, such as macular retinae, breast cancer, prostate cancer, leukemia, coronary heart disease, obesity, diabetes, schizophrenia, and arthritis $[13,14]$. These results have been published on many prestigious journals, in which a series of disease-related genes, genetic susceptibility regions and SNPs have been reported. As of December 2015, scientists have totally completed 1692 GWAS for 18 categories of nearly 300 complex diseases and traits, and have identified about 4000 SNPs that are associated with complex diseases and traits (Fig. 1).

\section{Application of GWAS to the study of CHB}

The first GWAS on CHB was published in 2009. As of February 2016, a total of eleven GWAS on CHB have been reported. In these studies, a number of CHBrelated susceptibility loci and genetic regions have been reported, among which 26 important candidate SNPs were identified (Table 1). As a newly emerging study tool, GWAS certainly needs to be further improved. The GWAS on CHB is recently getting started, therefore to summarize and properly interpret the GWAS data, and to understand the role of these loci in the pathogenesis of $\mathrm{CHB}$ in different populations will be critical for the application of GWAS to the study of CHB.

Scientists and scholars at home and abroad have recently performed a series of GWAS on $\mathrm{CHB}$ and found multiple CHB-related SNPs. In 2009, Kamatani first reported the GWAS on $\mathrm{CHB}$, in which he found two loci, rs9277535 within the HLA-DPB1 region and rs3077 within the HLA-DPA1 region, play important roles in the occurrence of CHB in Japanese population [15]. Following this study, Mbarek further confirmed Kamatani's

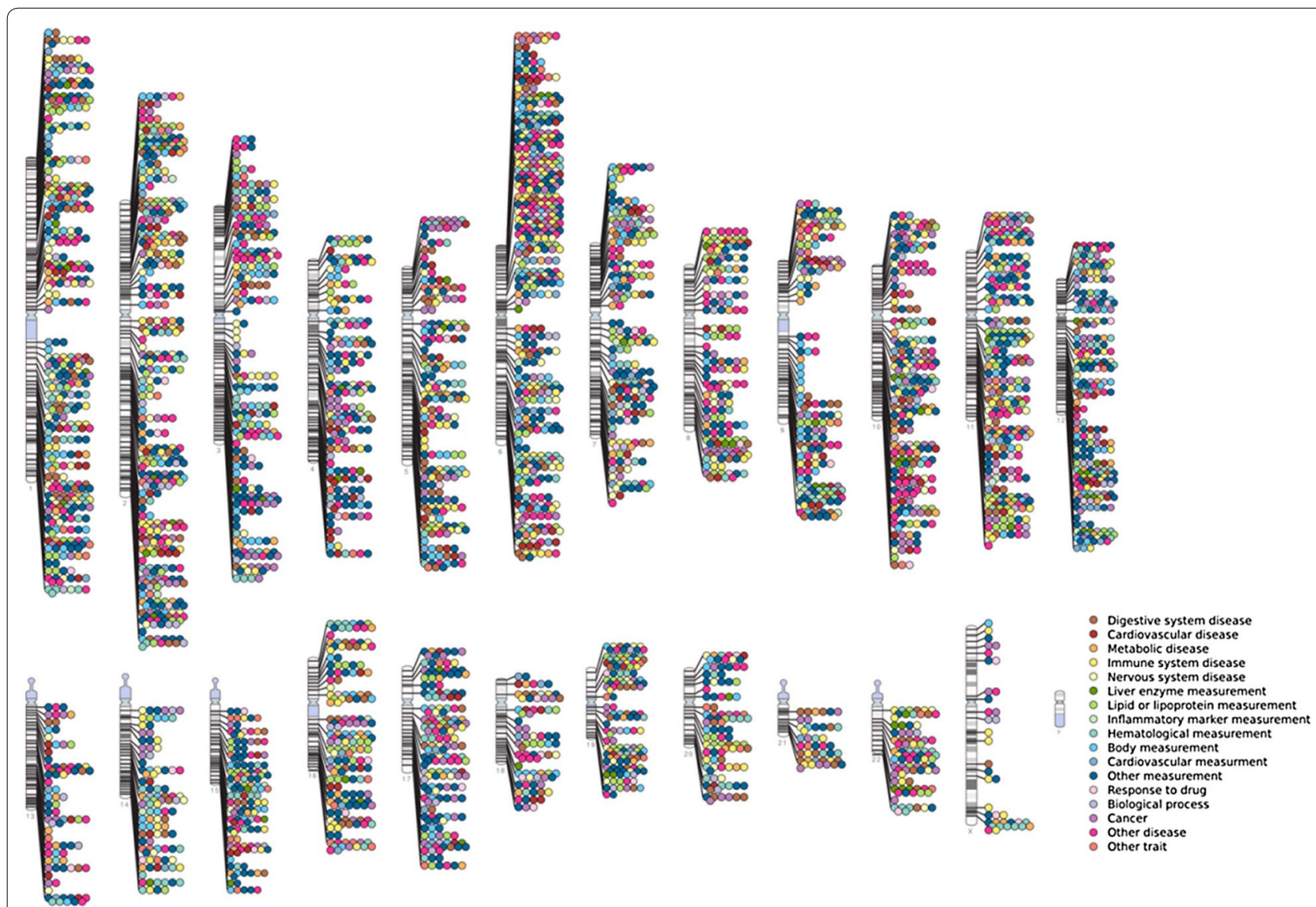

Fig. 1 The chromosomal distribution of SNPs associated with complex diseases and traits (NHGRI GWA Catalog: http://www.genome.gov/ GWAStudies) 
Table 1 GWAS on the genetic mechanism of chronic hepatitis B

\begin{tabular}{|c|c|c|c|c|c|c|}
\hline & Ethnicity & SNP & Chromosome (locus) & Odds ratio & $P$ value (combined stage) & References \\
\hline 1 & $\begin{array}{l}\text { Japanese } \\
\text { Korean } \\
\text { Indonesian }\end{array}$ & rs9277535 & $6(H L A-D P B 1)$ & $1.39-1.89$ & $\begin{array}{l}3.0 \times 10^{-54} \\
-3.0 \times 10^{-12}\end{array}$ & $\begin{array}{l}{[10-12]} \\
{[18,19]}\end{array}$ \\
\hline 2 & $\begin{array}{l}\text { Japanese } \\
\text { East Asian } \\
\text { Korean }\end{array}$ & rs3077 & 6 (HLA-DPA1) & $1.78-1.89$ & $\begin{array}{l}2.0 \times 10^{-61} \\
-2.0 \times 10^{-38}\end{array}$ & $\begin{array}{l}{[10-12]} \\
{[14,17,18]}\end{array}$ \\
\hline 3 & $\begin{array}{l}\text { Japanese } \\
\text { Korean }\end{array}$ & rs2856718 & 6 (TRNAI25) & $1.56-1.60$ & $\begin{array}{l}4.0 \times 10^{-37} \\
2.0 \times 10^{-24}\end{array}$ & $\begin{array}{l}{[11,12]} \\
{[17,18]}\end{array}$ \\
\hline 4 & $\begin{array}{l}\text { Japanese } \\
\text { Korean } \\
\text { Chinese }\end{array}$ & rs7453920 & $6(H L A-D Q B 2)$ & $1.81-2.00$ & $\begin{array}{l}5.0 \times 10^{-37} \\
\sim 7.0 \times 10^{-26}\end{array}$ & $\begin{array}{l}{[11,12,16]} \\
{[17,18]}\end{array}$ \\
\hline 5 & Korean & rs1419881 & 6 (TCF19) & 1.37 & $1.0 \times 10^{-18}$ & {$[12,18]$} \\
\hline 6 & Korean & rs652888 & 6 (EHMT2) & 1.38 & $7.0 \times 10^{-13}$ & {$[12,18]$} \\
\hline 7 & Korean & rs9394021 & 6 (VARS2) & 0.78 & $1.7 \times 10^{-6}$ & [13] \\
\hline 8 & Korean & rs2517459 & 6 (VARS2-SFTA2) & 0.56 & $1.7 \times 10^{-10}$ & [13] \\
\hline 9 & East Asian & rs9277542 & $6(H L A-D P B 1)$ & 1.64 & $2.0 \times 10^{-21}$ & [14] \\
\hline 10 & Chinese & rs11866328 & 16 (GRIN2A) & 1.68 & $2.0 \times 10^{-8}$ & [15] \\
\hline 11 & Chinese & rs4821116 & 22 (UBE2L3) & 1.22 & $2.0 \times 10^{-12}$ & [16] \\
\hline 12 & Chinese & rs3130542 & 6 (TRNAI25) & 1.33 & $9.0 \times 10^{-14}$ & {$[16,18]$} \\
\hline 13 & Chinese & rs352140 & 3 (TLR9) & 0.70 & $8.8 \times 10^{-3}$ & [17] \\
\hline 14 & Chinese & rs16944 & 2 (IL1B) & 0.67 & $1.6 \times 10^{-2}$ & [17] \\
\hline 15 & Chinese & rs3212227 & 5 (IL12B) & 1.38 & $2.1 \times 10^{-2}$ & [17] \\
\hline 16 & Chinese & rs3799488 & 13 (IFNGR1) & 1.48 & $4.8 \times 10^{-3}$ & [17] \\
\hline 17 & Chinese & rs1059293 & 6 (IFNGR2) & 0.27 & $1.1 \times 10^{-2}$ & [17] \\
\hline 18 & Chinese & rs467960 & $21(M \times 1)$ & 0.68 & $2.2 \times 10^{-2}$ & [17] \\
\hline 19 & Chinese & rs12614 & 6 (CFB) & 1.89 & $1.28 \times 10^{-34}$ & [18] \\
\hline 20 & Chinese & rs422951 & 6(NOTCH4) & 1.27 & $5.33 \times 10^{-16}$ & [18] \\
\hline 21 & Chinese & rs378352 & 6 (HLA-DOA) & 1.26 & $1.04 \times 10^{-23}$ & [18] \\
\hline 22 & Chinese & rs2853953 & $6(\mathrm{HLA}-\mathrm{C})$ & 1.47 & $5.06 \times 10^{-20}$ & [18] \\
\hline 23 & Chinese & rs1883832 & 20 (CD40) & 1.21 & $2.95 \times 10^{-15}$ & [18] \\
\hline 24 & Indonesian & rs9267665 & $6(H L A)$ & 2.05 & $1.0 \times 10^{-17}$ & [19] \\
\hline 25 & Chinese & rs477515 & 6 (TRNAI25) & 2.05 & $3.0 \times 10^{-19}$ & {$[20]$} \\
\hline 26 & $\begin{array}{l}\text { Indonesian } \\
\text { Chinese }\end{array}$ & rs3135363 & 6 (HLA-DR) & $1.53,1.51$ & $\begin{array}{l}7.0 \times 10^{-22} \\
8.0 \times 10^{-7}\end{array}$ & {$[19,20]$} \\
\hline
\end{tabular}

findings using GWAS also done with Japanese population [16]. Moreover, he and his colleagues found rs2856718-A within HLA-DQB1 region and rs7453920-G within HLADQB2 region are associated with the occurrence of $\mathrm{CHB}$. In 2013, Kim [17] completed the GWAS on CHB using South Korea population, and found that rs652888 in the gene EHMT2, and rs1419881 in the gene TCF19 within the HLA region, are associated with an increased risk of $\mathrm{CHB}$ occurrence. In addition, this group also verified that these loci-rs9277535, rs3077, rs7453920, and rs2856718 are all associated with the occurrence of $\mathrm{CHB}$ in both Japanese population and Korean population. Cheong et al. [18] recently found that rs9394021 and rs2517459 two SNP loci in the VARS2-SFTA2 gene region are the new genetic markers in the Korean population of $\mathrm{CHB}$ in 2015. Nishida N conducted the first GWAS on how to protect from hepatitis B and clear HBV [19]. It was found that rs3077 in HLA-DPA1 region and rs9277542 in HLADPB1 region, both have a protective effect on $\mathrm{CHB}$ in East Asian population, and both are associated with $\mathrm{HBV}$ clearance.

Chinese scholars Liu L et al. first conducted GWAS on CHB with Chinese Han population in 2011 and found that $\mathrm{rs} 11866328 \mathrm{G}$, located in the GRIN2A gene within 16 13.2 region, is a susceptibility locus associated with disease progression in carriers of HBV [20]. Recently $\mathrm{Hu}$ $\mathrm{Z}$ and his colleagues found that rs3130542-A in the gene TRNAI25 within 6p21.33 (near the HLA-C) region, and rs4821116-G in the gene UBE2L3 within the $22 \mathrm{q} 11.21$ region, are both associated with the occurrence of $\mathrm{CHB}$ 
in Chinese Han population [21]. Moreover, they further confirmed that rs7453920 is associated with the occurrence of $\mathrm{CHB}$ in Japanese and Korean populations as previously reported; they also found that this locus is actually associated with high prevalence of $\mathrm{CHB}$ in Chinese Han population. in 2015, a GWAS study on CHB performed by He et al. [22] in southwest China revealed 6 novel loci associated with increased prevalence rate of chronic hepatitis $B$, which are rs 352140 on the gene TLR9, rs16944 on the gene IL1B, rs3212227 on the gene IL12B, rs3799488 on the gene IFNGR1, rs1059293 on the gene IFNGR2, rs467960 on the gene MX1, respectively. In addition, they also confirmed that the previously discovered 4 SNP loci namely rs3077, rs2856718, rs9277535, and rs7453920, are associated with CHB in this area. In the same year, another GWAS study led by Jiang et al. [23] in the eastern part of China revealed 5 novel CHB susceptibility loci, including rs12614 on the gene $\mathrm{CFB}, \mathrm{rs} 422951$ on the gene NOTCH4, rs378352 on the gene HLA-DOA, rs188332 on the gene HLA-C, and rs 18838325 on the gene CD40, respectively. In 2016, Zhu et al. fine mapped the histocompatibility complex $(\mathrm{MHC})$ region by using their existing GWAS data and identified four additional susceptibility loci that independently drove the chronic HBV infection in Han Chinese [24]. Nishida et al. applied HLA imputation method to determine HLA alleles by using genome-wide SNP typing data of 1975 Japanese individuals, and found that a SNP located in the HLA-DP locus from GWAS was strongly associated with $\mathrm{CHB}$ susceptibility [25]. Xiang et al. found that human leukocyte antigen DP/DQ gene (HLA-DP/DQ) polymorphisms (rs9277471, rs9277535, and rs9277542 in HLA-DP; rs9272346 in HLA-DQ) are associated with chronic hepatitis B in Chinese Han (400 patients) and Uygur (399 patients) populations [26]. In 2017, Shin et al. identified rs1265163 in OCT4 as a novel genetic marker for CHB susceptibility in a 3902 Korean individuals in a follow-up study to their GWAS [27].

In order to evaluate the immune response after injection of hepatitis B vaccine, Png E and his colleagues completed in 2011 the first GWAS on the immune response of hepatitis B vaccine in the population of Indonesia [28]. It was found that rs3135363 within the HLA-DR region, rs9277535 within the HLA-DPB1 region, and rs9267665 within the HLA-DP region are all associated with an immune response after the injection of hepatitis $B$ vaccine. A subsequent GWAS completed by Pan also confirmed that rs3135363 is associated with an immune response after injection of hepatitis B vaccine in the Chinese Han population [29]. However, the locus rs477515$\mathrm{T}$ located within the gene TRNAI25, $15 \mathrm{~kb}$ upstream of HLA-DRB1, showed no significant correlation with the immune response of hepatitis $B$ vaccine.
The genetic background among human populations is generally different and the mechanism of pathogenesis can also differ. As a complex disease which involves multi-genes, chronic HBV infection is challenging to work on and the conclusions drew in the studies can be inconsistent due to the differences in the genetic background of populations and area studied. Therefore, the relationship between $\mathrm{CHB}$ and SNP loci screened by GWAS still remains unclear. More populations and larger independent samples are needed in order to improve the consistency of GWAS results.

\section{Conclusions}

At present, GWAS only analyzes the effect of a single locus on the disease susceptibility. However, such effect on the complex disease is generally very weak, therefore this strategy cannot be simply taken to investigate the causes of complex diseases. Hence, how to find an effective way to perform in-depth analysis for GWAS data and then detect more susceptibility genes has become a new research hotspot [30]. To date, different strategies and approaches have been successively taken in the follow-up studies of GWAS for complex diseases in order to perform in-depth data mining. For example, when testing the correlation between a SNP and certain diseases, the interactions between this SNP and other SNPs will be also thoroughly examined (i.e., epistatic effect). Several analytical methods, such as Bayesian epistasis, classification and regression trees, and multifactor dimensionality reduction, have been widely used in data mining [31-33]. The application of these strategies and approaches has compensated for the disadvantage of classical GWAS, and has also deepened our understanding in the genetic mechanisms of complex human diseases. For this reason, the follow-up studies for the GWAS data are therefore of high importance.

\section{Abbreviations \\ CHB: chronic hepatitis B; HBV: hepatitis B virus; GWAS: genome-wide associa- tion study.}

\section{Authors' contributions}

$B Q$ and WJ initiated the review; $B Q, W J, A M, H H, Y Z$, and LT performed the review, analyzed the data, and wrote the manuscript; $\mathrm{MO}$ and $\mathrm{KS}$ reviewed and commented on the manuscript, and provided final approval. All authors read and approved the final manuscript.

\footnotetext{
Author details

1 Department of Gastroenterology, Heilongjiang Province Hospital, 82 Zhongshan Road, Harbin 150036, Heilongjiang, People's Republic of China. ${ }^{2}$ Department of Gastroenterology, The First Affiliated Hospital of Jiamusi University, Jiamusi 154002, People's Republic of China. ${ }^{3}$ Division of Gastroenterology, Department of Internal Medicine, University of Kansas, Medical Center, Kansas City 66160, USA. ${ }^{4}$ Department of Gastroenterology, Asahi General Hospital, Chiba 289-2511, Japan. ${ }^{5}$ Department of Laboratory Diagnosis, Heilongjiang Province Hospital, Harbin 150036, People's Republic of China.
} 


\section{Acknowledgements}

The authors are grateful to Mojtaba Olyaee (Department of Gastroenterology and Hepatology, the University of Kansas Hospital, Kansas, America), Kenji Shimura and Akihiro Miyakawa (Department of gastroenterology, Asahi General Hospital, Chiba, Japan) for providing helpful suggestions, data analysis, and linguistic modification.

\section{Competing interests}

The authors declare that they have no competing interests.

\section{Availability of data and materials}

The datasets are available from the corresponding author under reasonable request.

\section{Consent for publication}

All authors were informed and agreed to publish this work under the current authorship.

\section{Ethics approval and consent to participate}

The Ethics Committee of Harbin Medical University approved this study. Consent to participate was not applicable.

\section{Funding}

This work was supported by the Postdoctoral Research Start-up Fund of Heilongjiang Province in China (grant no. LBH-Q17160) and the Foundation of Excellent Returned Overseas Students of Heilongjiang Province in China (grant no. 2015-424)

\section{Publisher's Note}

Springer Nature remains neutral with regard to jurisdictional claims in published maps and institutional affiliations.

Received: 1 October 2016 Accepted: 1 November 2017 Published online: 28 December 2017

\section{References}

1. Bhat M, Ghali P, Deschenes M, Wong P. Prevention and management of chronic hepatitis B. Int J Prev Med. 2014;5(Suppl 3):S200-7.

2. Liaw YF, Kao JH, Piratvisuth T, Chan HL, Chien RN, Liu CJ, Gane E, Locarnini S, Lim SG, Han KH, Amarapurkar D, Cooksley G, Jafri W, Mohamed R, Hou $J$, Chuang WL, et al. Asian-Pacific consensus statement on the management of chronic hepatitis B: a 2012 update. Hep Int. 2012:6(3):531-61.

3. Trepo C, Chan HL, Lok A. Hepatitis B virus infection. Lancet. 2014:384(9959):2053-63.

4. Cui Y, Jia J. Update on epidemiology of hepatitis B and C in China. J Gastroenterol Hepatol. 2013;28(Suppl 1):7-10.

5. Ezzikouri S, Ozawa M, Kohara M, Elmdaghri N, Benjelloun S, TsukiyamaKohara K. Recent insights into hepatitis B virus-host interactions. J Med Virol. 2014;86(6):925-32

6. Thorat M. Tuning into the genetic orchestra using microarrays: limitations of DNA microarrays in clinical practice. Nat Clin Pract Oncol. 2006;3(12):E1.

7. Lacey S, Chung JY, Lin H. A comparison of whole genome sequencing with exome sequencing for family-based association studies. BMC Proc. 2014;8(Suppl 1 Genetic Analysis Workshop 18Vanessa Olmo):S38.

8. Lalonde E, Albrecht S, Ha KC, Jacob K, Bolduc N, Polychronakos C, Dechelotte P, Majewski J, Jabado N. Unexpected allelic heterogeneity and spectrum of mutations in Fowler syndrome revealed by next-generation exome sequencing. Hum Mutat. 2010;31(8):918-23.

9. Petersen BS, Fredrich B, Hoeppner MP, Ellinghaus D, Franke A. Opportunities and challenges of whole-genome and -exome sequencing. BMC Genet. 2017;18(1):14.

10. Kim SY, Li Y, Guo Y, Li R, Holmkvist J, Hansen T, Pedersen O, Wang J, Nielsen R. Design of association studies with pooled or un-pooled next-generation sequencing data. Genet Epidemiol. 2010;34(5):479-91.

11. Seal A, Gupta A, Mahalaxmi M, Aykkal R, Singh TR, Arunachalam V. Tools, resources and databases for SNPS and indels in sequences: a review. Int J Bioinform Res Appl. 2014;10(3):264-96.
12. Klein RJ, Zeiss C, Chew EY, Tsai JY, Sackler RS, Haynes C, Henning AK, SanGiovanni JP, Mane SM, Mayne ST, Bracken MB, Ferris FL, Ott J, Barnstable C, Hoh J. Complement factor $\mathrm{H}$ polymorphism in age-related macular degeneration. Science. 2005;308(5720):385-9.

13. Arking D, Rommens J. Editorial overview: molecular and genetic bases of disease: Enter the post-GWAS era. Curr Opin Genet Dev. 2015;33:77-9.

14. van der Sijde MR, Ng A, Fu J. Systems genetics: from GWAS to disease pathways. Biochem Biophys Acta. 2014;1842(10):1903-9.

15. Kamatani Y, Wattanapokayakit S, Ochi H, Kawaguchi T, Takahashi A, Hosono N, Kubo M, Tsunoda T, Kamatani N, Kumada H, Puseenam A, Sura T, Daigo Y, Chayama K, Chantratita W, Nakamura Y, et al. A genome-wide association study identifies variants in the HLA-DP locus associated with chronic hepatitis B in Asians. Nat Genet. 2009;41(5):591-5.

16. Mbarek H, Ochi H, Urabe Y, Kumar V, Kubo M, Hosono N, Takahashi A, Kamatani Y, Miki D, Abe H, Tsunoda T, Kamatani N, Chayama K, Nakamura Y, Matsuda K. A genome-wide association study of chronic hepatitis B identified novel risk locus in a Japanese population. Hum Mol Genet. 2011;20(19):3884-92

17. Kim YJ, Kim HY, Lee JH, Yu SJ, Yoon JH, Lee HS, Kim CY, Cheong JY, Cho SW Park NH, Park BL, Namgoong S, Kim LH, Cheong HS, Shin HD. A genomewide association study identified new variants associated with the risk of chronic hepatitis B. Hum Mol Genet. 2013;22(20):4233-8.

18. Cheong HS, Lee JH, Yu SJ, Yoon JH, Lee HS, Cheong JY, Cho SW, Park NH, Park BL, Namgoong S, Kim LH, Shin HD, Kim YJ. Association of VARS2SFTA2 polymorphisms with the risk of chronic hepatitis B in a Korean population. Liver Int. 2015;35(8):1934-40.

19. Nishida N, Sawai H, Matsuura K, Sugiyama M, Ahn SH, Park JY, Hige S, Kang JH, Suzuki K, Kurosaki M, Asahina Y, Mochida S, Watanabe M, Tanaka E, Honda M, Kaneko S, et al. Genome-wide association study confirming association of HLA-DP with protection against chronic hepatitis B and viral clearance in Japanese and Korean. PLOS ONE. 2012:7(6):e39175.

20. Liu L, Li J, Yao J, Yu J, Zhang J, Ning Q, Wen Z, Yang D, He Y, Kong X, Song $\mathrm{Q}$. Chen $\mathrm{M}$, Yang $\mathrm{H}$, Liu Q, Li S, Lin J. A genome-wide association study with DNA pooling identifies the variant rs11866328 in the GRIN2A gene that affects disease progression of chronic HBV infection. Viral Immunol. 2011:24(5):397-402

21. Hu Z, Liu Y, Zhai X, Dai J, Jin G, Wang L, Zhu L, Yang Y, Liu J, Chu M, Wen J, Xie K, Du G, Wang Q, Zhou Y, Cao M, et al. New loci associated with chronic hepatitis B virus infection in Han Chinese. Nat Genet. 2013:45(12):1499-503.

22. He D, Tao S, Guo S, Li M, Wu J, Huang H, Guo X, Yan G, Zhu P, Wang Y. Interaction of TLR-IFN and HLA polymorphisms on susceptibility of chronic HBV infection in Southwest Han Chinese. Liver Int. 2015;35(8):1941-9.

23. Jiang DK, Ma XP, Yu H, Cao G, Ding DL, Chen $H$, Huang HX, Gao YZ, Wu $X P$, Long XD, Zhang H, Zhang Y, Gao Y, Chen TY, Ren WH, Zhang P, et al. Genetic variants in five novel loci including CFB and CD40 predispose to chronic hepatitis B. Hepatology. 2015;62(1):118-28.

24. Zhu M, Dai J, Wang C, Wang Y, Qin N, Ma H, Song C, Zhai X, Yang Y, Liu J, Liu L, Li S, Yang H, Zhu F, Shi Y, Shen H, et al. Fine mapping the MHC region identified four independent variants modifying susceptibility to chronic hepatitis B in Han Chinese. Hum Mol Genet. 2016;25(6):1225-32.

25. Nishida N, Ohashi J, Khor SS, Sugiyama M, Tsuchiura T, Sawai H, Hino K, Honda M, Kaneko S, Yatsuhashi H, Yokosuka O, Koike K, Kurosaki M, Izumi $\mathrm{N}$, Korenaga M, Kang JH, et al. Understanding of HLA-conferred susceptibility to chronic hepatitis B infection requires HLA genotyping-based association analysis. Sci Rep. 2016;6:24767.

26. Xiang X, Guo Y, Yang L, Ge Q, Mijit S, Xu F. Association of human leukocyte antigen DP/DQ gene polymorphisms with chronic hepatitis B in Chinese Han and Uygur populations. Infect Genet Evol J Mol Epidemiol Evolut Genet Infect Dis. 2016:43:407-11.

27. Shin JG, Cheong HS, Lee K, Ju BG, Lee JH, Yu SJ, Yoon JH, Cheong JY, Cho SW, Park NH, Namgoong S, Kim LH, Kim YJ, Shin HD. Identification of novel OCT4 genetic variant associated with the risk of chronic hepatitis $B$ in a Korean population. Liver Int. 2017:37(3):354-61.

28. Png E, Thalamuthu A, Ong RT, Snippe H, Boland GJ, Seielstad M. A genome-wide association study of hepatitis $B$ vaccine response in an Indonesian population reveals multiple independent risk variants in the HLA region. Hum Mol Genet. 2011;20(19):3893-8.

29. Pan L, Zhang L, Zhang W, Wu X, Li Y, Yan B, Zhu X, Liu X, Yang C, Xu J, Zhou G, Xu A, Li H, Liu Y. A genome-wide association study identifies polymorphisms in the HLA-DR region associated with non-response to 
hepatitis B vaccination in Chinese Han populations. Hum Mol Genet. 2014;23(8):2210-9.

30. Begum F, Sharker MH, Sherman SL, Tseng GC, Feingold E. Regionally Smoothed Meta-Analysis Methods for GWAS Datasets. Genet Epidemiol. 2016;40(2):154-60.

31. MakTS, Best N, Rushton L. Robust bayesian sensitivity analysis for casecontrol studies with uncertain exposure misclassification probabilities. Int J Biostat. 2015;11(1):135-49.
32. Henrard S, Speybroeck N, Hermans C. Classification and regression tree analysis vs. multivariable linear and logistic regression methods as statistical tools for studying haemophilia. Haemophilia. 2015;21(6):715-22.

33. Pan Q, Hu T, Moore JH. Epistasis, complexity, and multifactor dimensionality reduction. Methods Mol Biol. 2013;1019:465-77.

\section{Submit your next manuscript to BioMed Central and we will help you at every step:}

- We accept pre-submission inquiries

- Our selector tool helps you to find the most relevant journal

- We provide round the clock customer support

- Convenient online submission

- Thorough peer review

- Inclusion in PubMed and all major indexing services

- Maximum visibility for your research

Submit your manuscript at www.biomedcentral.com/submit 